\title{
The Magnitude of Time-Dependent Bias in the Estimation of Excess Length of Stay Attributable to Healthcare-Associated Infections
}

\author{
Richard E. Nelson, PhD; $;^{1,2}$ Scott D. Nelson, PharmD; ${ }^{1,3}$ Karim Khader, PhD; ${ }^{1,2}$ Eli L. Perencevich, MD, MS; ${ }^{4,5}$ \\ Marin L. Schweizer, PhD; ${ }^{4,5}$ Michael A. Rubin, MD, PhD; ${ }^{1,2}$ Nicholas Graves, PhD; ${ }^{6}$ Stephan Harbarth, MD, MS; ${ }^{7}$ \\ Vanessa W. Stevens, PhD; ${ }^{1,3}$ Matthew H. Samore, $\mathrm{MD}^{1,2}$
}

\begin{abstract}
BACKGROUND. Estimates of the excess length of stay (LOS) attributable to healthcare-associated infections (HAIs) in which total LOS of patients with and without HAIs are biased because of failure to account for the timing of infection. Alternate methods that appropriately treat HAI as a time-varying exposure are multistate models and cohort studies, which match regarding the time of infection. We examined the magnitude of this time-dependent bias in published studies that compared different methodological approaches.
\end{abstract}

метнодs. We conducted a systematic review of the published literature to identify studies that report attributable LOS estimates using both total LOS (time-fixed) methods and either multistate models or matching patients with and without HAIs using the timing of infection.

RESULTS. Of the 7 studies that compared time-fixed methods to multistate models, conventional methods resulted in estimates of the LOS to HAIs that were, on average, 9.4 days longer or $238 \%$ greater than those generated using multistate models. Of the 5 studies that compared timefixed methods to matching on timing of infection, conventional methods resulted in estimates of the LOS to HAIs that were, on average, 12.6 days longer or $139 \%$ greater than those generated by matching on timing of infection.

CONCLUSION. Our results suggest that estimates of the attributable LOS due to HAIs depend heavily on the methods used to generate those estimates. Overestimation of this effect can lead to incorrect assumptions of the likely cost savings from HAI prevention measures.

Infect. Control Hosp. Epidemiol. 2015;36(9):1089-1094

Healthcare-associated infections (HAIs) are an important patient safety component of several key pieces of healthcare legislation in the United States. In 2008, the Centers for Medicare and Medicaid Services stopped reimbursing hospitals for several HAIs classified as "never events." Additionally, as part of the Affordable Care Act (ACA), the hospital-acquired condition (HAC) reduction program imposes financial penalties for hospitals that perform poorly on measures of hospitalacquired adverse events, including HAIs. ${ }^{2}$

The measurement of the cost savings associated with interventions to decrease the rates of HAIs is becoming an important tool for evaluating the cost-effectiveness of such policies. An important component of hospital costs attributable to HAI is the excess length of hospital stay (LOS).

Estimating the impact of HAI on LOS, however, is challenging because HAIs, by definition, are not present during a patient's entire hospitalization. ${ }^{3,4}$ Only those hospital days that accrue after the occurrence of the nosocomial infection are possibly secondary to infection. Van Walraven et $\mathrm{al}^{5}$ claimed, and Beyersmann et $\mathrm{al}^{6}$ provided mathematical proof, that treating a time-dependent exposure as time-fixed inflates estimates of the effect of that exposure on an outcome whether (1) the true effect prolongs the outcome, (2) the true effect accelerates the outcome, or (3) the exposure has no true effect on the outcome. For example, estimates of the excess LOS due to an HAI that do not take into account the fact that the HAI is not present during a patient's entire hospital stay always overestimate the effect, regardless of what the true effect is. The concept of time-dependent bias is well appreciated, but less is known about the magnitude of its impact, particularly as it relates to outcomes attributable to HAIs. ${ }^{7}$

The aim of this analysis was to summarize and quantify findings of studies of HAI-attributable LOS that compared

Affiliations: 1. Veterans Affairs Salt Lake City Health Care System, Salt Lake City, Utah, United States; 2. Department of Internal Medicine, University of Utah School of Medicine, Salt Lake City, Utah, United States; 3. Department of Pharmacotherapy, University of Utah College of Pharmacy, Salt Lake City, Utah, United States; 4. Iowa City Veterans Affairs Health Care System, Iowa City, Iowa, United States; 5. Department of Internal Medicine, University of Iowa Carver College of Medicine, Iowa City, Iowa, United States; 6. School of Public Health and Social Work, Queensland University of Technology, Brisbane, Australia; 7. Infection Control Program, University of Geneva Hospitals and Faculty of Medicine, Geneva, Switzerland.

The views expressed in this article are those of the authors and do not necessarily reflect the position or policy of the Department of Veterans Affairs or the United States government. Received November 21, 2014; accepted April 22, 2015; electronically published June 4, 2015

(c) 2015 by The Society for Healthcare Epidemiology of America. All rights reserved. 0899-823X/2015/3609-0013. DOI: 10.1017/ice.2015.129 
methods that accounted for the timing of infection with those that do not account for the timing of infection. The purpose of this comparison is to inform the interpretation of literature on cost-saving impacts of preventing HAIs. An estimation of the magnitude of bias may be useful for decision analytic scientists to deflate the cost and LOS input parameters in costeffectiveness analyses. The demonstration that this bias leads to nontrivial excess LOS estimates may also guide investigators when choosing between available epidemiologic study designs and statistical methods. Ultimately, policy makers may have more reasonable expectations regarding the effect of infection prevention efforts.

\section{Time-Fixed Methods for Estimating Excess LOS Due to HAI}

Estimates of excess LOS due to HAI are often generated by comparing the total LOS between patients who acquire HAIs during their hospitalization and those who do not. ${ }^{8-11}$ This comparison can be conducted as a simple comparison of means, as a multivariable regression model, or even using propensity score matching. However, because none of these methods accounts for the time-varying nature of the HAI, they are all subject to time-dependent bias. This bias occurs because in methods that treat the HAI as time fixed, the time spent in the hospital prior to the occurrence of the HAI is incorrectly attributed to the HAI. Therefore, the pre-HAI LOS will be added to the post-HAI LOS, thus making the attributable LOS appear to be larger than it actually is. In addition, patients are at greater risk for HAIs the longer they are in the hospital. This reverse causality can also lead to biased estimates of the true effect of HAI on LOS.

\section{Methods for Overcoming Time-Dependent Bias}

Two primary methods for overcoming time-dependent bias have been utilized in published studies: multistate model and matching on timing of infection. Both methods accomplish this task by taking into account the timing of infection. Multistate models treat an HAI as one of several mutually exclusive states through which a hospitalized patient can move. The method of matching on the timing of infection matches patients with HAIs to patients without HAIs with a requirement that the non-HAI patient LOS be at least as long as the time until the HAI in the infected case patient. In performing this matching, it is important that the selected uninfected patients are those who could still become infected at a later date. This method, called exposure density sampling, has been shown to reduce bias in the estimation of the effect of HAI because it is not conditional based on a future event. ${ }^{12}$ By conditioning on the future event of having an HAI or not at a later date, matching methods that do not employ exposure density sampling are subject to similar biases that plague conventional estimation methods that do not take the timing of infection into account.
In the following section, we summarize the studies identified through a systematic literature search of PubMed for combinations of the following search terms: time-dependent bias, length of stay, attributable length of stay, excess length of stay, hospital-acquired infection, healthcare-associated infection, and nosocomial infection. From the list of published papers identified through these searches, we chose those that used one or both of these methods as well as methods in which an HAI was treated as a time-fixed variable using the same cohort of patients.

\section{RES ULTS}

\section{Search}

Our literature search yielded 516 records; of these, 276 were duplicates. Of the 240 articles that remained after the removal of duplicates, 8 met the criteria described above and were included in our analysis and summary. To overcome timedependent bias in the estimation of the excess LOS associated with HAIs, 4 studies used multistate models only, 2 studies matched HAI patients to control patients on the timing of infection, and 2 studies used both methods.

\section{Multistate Models}

(Table 1) In a landmark paper in 2000, Schulgen ${ }^{13}$ illustrated these concepts using 2 separate studies. The first study (Study 1) consisted of 765 patients admitted to the intensive care unit (ICU) after having undergone open heart, large-bowel, or biliary-tract surgery between November 1988 and September 1989. In Study 2, 756 patients were followed who were admitted to anesthesiological or medical ICUs in the same hospital between July 1991 and July 1993. The authors used 2 methods to estimate the impact of HAI on LOS in which the HAI was treated as a time-fixed variable. The first was a simple comparison of the unadjusted mean LOS for patients with and without an HAI. This second method involved a comparison of mean LOS after matching patients with and without an HAI on observable baseline confounders such as age, sex, diagnosis, and, for Study 1 only, the degree of contamination of the surgical wound. The multistate model used by Schulgen ${ }^{13}$ included 5 states: no nosocomial infection, nosocomial pneumonia, nosocomial sepsis, discharge, and death.

Roberts et $\mathrm{al}^{14}$ examined outcomes for a cohort of 1,500 patients admitted to a large teaching hospital in the United States in the year 2000. Of these patients, 159 (10.6\%) developed an HAI during their hospitalization. Treating HAI as a timefixed variable, an ordinary least squares regression was used to estimate the impact of HAI on LOS. To overcome timedependent bias, the investigators used multistate model with 3 states: no HAI, HAI, and discharge/death.

Using data from 9,545 patients (8.7\% of whom had HAIs) admitted to the ICU in 1 of 11 hospitals in Buenos Aires, Argentina, Barnett et $\mathrm{al}^{3}$ estimated the impact of HAI on LOS 
TABLE 1. Published Estimates of the Magnitude of Time-Dependent Bias: Conventional Methods vs Multistate Models

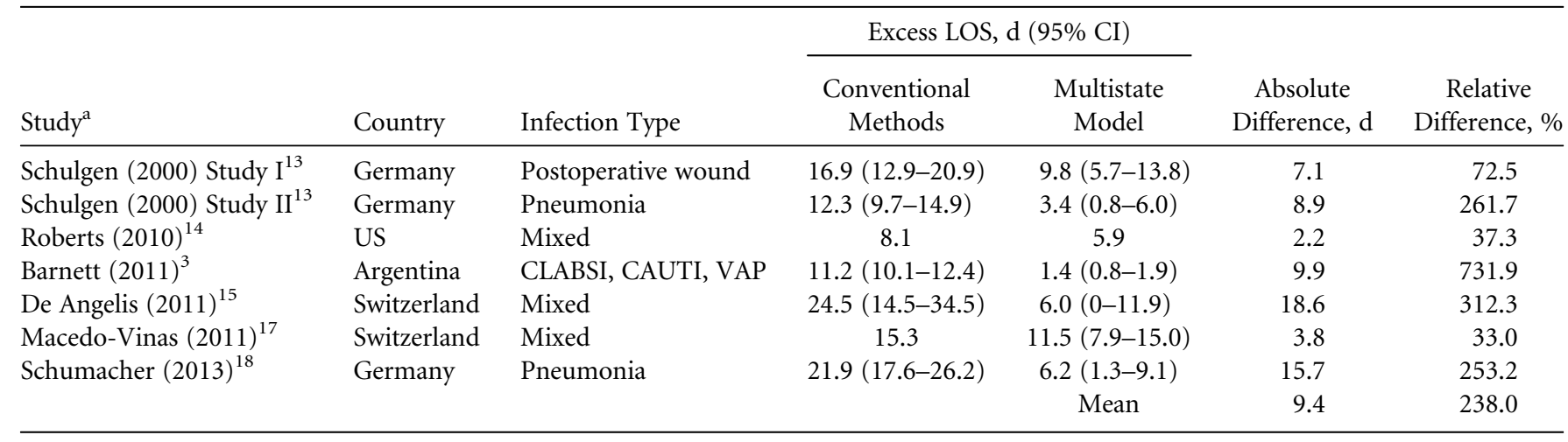

NOTE. HAI, healthcare-associated infection; LOS, length of stay; CI, confidence interval; CLABSI, central line associated bloodstream infection; CAUTI, catheter-associated urinary tract infection; VAP, ventilator associated pneumonia.

${ }^{a}$ De Angelis (2011) and Macedo-Vinas (2011) stated that they identified HAIs due to methicillin-resistant Staphylococcus aureus. None of the other studies explicitly stated which organism was associated with the HAIs.

тавцE 2. Published Estimates of the Magnitude of Time-Dependent Bias: Conventional Methods vs Matching on Timing of Infection

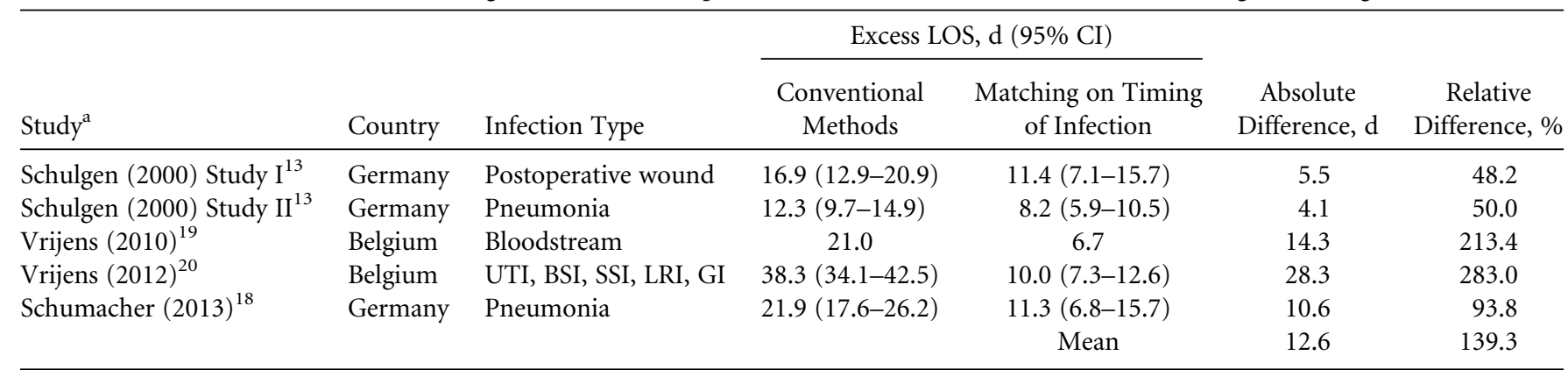

NOTE. HAI, healthcare-associated infection; LOS, length of stay; CI, confidence interval; UTI, urinary tract infection; BSI, bloodstream infection; SSI, surgical site infection; GI, gastrointestinal infection.

${ }^{a}$ None of the studies explicitly stated which organism was associated with the HAIs.

assuming time-fixed HAI using a parametric $\gamma$ model. This method yielded an excess LOS that was substantially larger than the estimate generated using a 3-state (no HAI, HAI, discharge/death) multistate model. The $700 \%$ relative difference between the 2 results was the largest relative difference of any among the studies in this analysis.

De Angelis et $\mathrm{al}^{15}$ used data from a prospective cohort study ${ }^{16}$ of 976 patients admitted to the University of Geneva Hospital between July 2004 and May 2006, with 167 having a nosocomial MRSA infection. Wolkewitz et $\mathrm{al}^{16}$ used a fourth state in their multistate model that captured MRSA colonization as an intermediate state between admission and MRSA infection or admission and discharge/death. The estimates generated using methods that treated HAI as a time-fixed exposure are reported in the subsequent paper by Wolkewitz et al. ${ }^{16}$

Using data from surgery patients admitted to the University of Geneva Hospital during 2009, Macedo-Vinas et $\mathrm{al}^{17}$ estimated the excess LOS due to MRSA HAI using a multistate model with data from 167 infected patients and 25,766 uninfected patients. This estimate was slightly lower than the estimate obtained by taking the difference in mean LOS between the infected patients and a cohort of uninfected patients who were matched 2:1 (uninfected to infected) on age, sex, and diagnosis-related group.

Schumacher et $\mathrm{al}^{18}$ used data from a university hospital in Germany. Of the 1,876 patients admitted to the ICU during 2000-2001, 158 developed nosocomial pneumonia. These investigators contrast the excess LOS obtained using a Cox proportional hazards regression model with those from a multistate model with 4 states: no HAI, HAI, discharge, and death.

\section{Matching on the Timing of Infection}

In addition to a multistate model described in the earlier section, Schulgen also matched infected and non-infected patients on time to infection (Table 2). ${ }^{13}$ The noninfected patients eligible to be matched were those who were discharged from the hospital without infections so exposure density sampling was not used. This method yielded estimates of the excess LOS that were larger than those generated from multistate models. 
Vrijens et $\mathrm{al},{ }^{19}$ with a cohort of 1,839 patients admitted to 1 of 19 acute care hospitals in Belgium, examined the impact of bloodstream HAIs on LOS. ${ }^{19}$ Without taking the timing of infection into account, the investigators matched each infected patient to 1 noninfected patient regarding age, all-patient refined-diagnosis-related group (APR-DRG), principal diagnosis, and Charlson comorbidity index. The attributable LOS was calculated as the mean difference in LOS between the infected and matched uninfected patients. An additional analysis added the time of infection as a matching criterion. The authors do not state whether exposure density sampling was used.

In 2012, Vrijens et al ${ }^{20}$ extended their earlier work by including urinary tract, surgical site, and gastrointestinal infections along with bloodstream infections from 659 patients in 63 acute care hospitals in Belgium. These investigators demonstrated the importance of including the time prior to infection, which they refer to as exposure duration, as a matching variable when selecting noninfected control patients and matched infected and noninfected patients in a 3:1 ratio. The absolute difference in excess LOS estimates from matching on hospital, APR-DRG, and age compared to matching on these 3 variables plus exposure duration was 28.3 days, the highest absolute difference of any study in this analysis. Again, there is no indication as to whether exposure density sampling was used.

Finally, in addition to the multistate model described above, Schumacher et $\mathrm{al}^{18}$ also matched their infected patients to uninfected patients. This matching used exposure density sampling, and a hazard ratio resulted from this exercise that was very similar to the one generated from the multistate model but with a wider confidence interval $(0.64 ; 95 \% \mathrm{CI}$, $0.55-0.73$ vs 0.65 days; $95 \%$ CI, $0.51-0.83)$. However, for this method, the excess LOS was not reported.

\section{Magnitude of Time-Dependent Bias}

These summary estimates indicate that estimates of bias were greater when time-fixed methods were compared to multistate models than when they were compared with matched cohort analyses. This is true even within the same studies. For example, in Schumacher et al, the absolute (relative) differences in estimates of excess LOS were 15.7 (253.2\%) when using a multistate model and $10.6(93.8 \%)$ when matching on the timing of infection. Similarly, in the 2 studies described in Schulgen et al, the multistate model yielded an absolute (relative) difference of excess LOS of 9.0-10.0 days (91.8\%$292.6 \%$ ) and matching on the timing of infection resulted in an absolute (relative) difference of excess LOS of 5.2-7.4 days $(62.7 \%-64.9 \%)$ compared with time-fixed approaches.

\section{I S C U S S I O N}

The results summarized here are useful for a number of purposes. First, with these estimates, policy and decision makers can make more realistic projections of the financial impact of infection prevention efforts. Second, when constructing models to perform cost-effectiveness analyses, analysts can use the estimates presented here to describe with greater accuracy the expected number of bed days saved from infection prevention programs. Not adjusting for sources of bias when estimating extra stay yields an overstatement of the cost savings from infection control programs and makes them appear to have greater value for money. Third, a demonstration that this bias leads to non-trivial difference in estimated LOS may further convince investigators of the importance of using alternative methods to the total LOS approach.

The magnitude of time-dependent bias in the estimation of excess LOS due to HAIs may depend on a number of factors, including the infection rate, the discharge rate, confounding, and model specification. In describing immortal time bias, Suissa ${ }^{21}$ determined that the magnitude of this type of bias depends on the proportion of the exposed group's time that is, in fact, unexposed (ie, immortal time). In other words, the immortal time bias is greater when more of the exposed patients' time is unexposed. Suissa also showed that the size of this bias depends positively on the proportion of unexposed to exposed person-time.

Overestimation of the attributable LOS caused by treating a time-varying exposure as if it was present at baseline can occur in any of the many possible hospital-acquired adverse events. ${ }^{22}$ Noninfectious disease-related events that are listed as part of the ACA HAC reduction program include pressure ulcers; iatrogenic pneumothorax; postoperative hip fractures, pulmonary embolism, and deep vein thrombosis; wound dehiscence; and accidental puncture and lacerations. ${ }^{23}$ The reduction program will no doubt lead to efforts to reduce these adverse events, and economic analyses will be needed to evaluate the impacts of these efforts. However, these analyses will not be as useful as they could be because of inaccurate inputs resulting from time-dependent bias. Better estimates of the impact of non-HAI hospital-acquired adverse events are clearly needed. ${ }^{21}$ In the meantime, the estimates of the magnitude of time-dependent bias presented here can be used to deflate estimates for other conditions.

Estimates of the size of time-dependent bias may vary based on many factors. For instance, the bias may be reduced when the HAI occurs early during the hospitalization (eg, early-onset pneumonia after major trauma). The severity and type of infection as well as the infectious pathogen may also play a role, such as how soon after admission the HAI occurs. Finally, time-dependent bias may not be relevant for studies investigating the inpatient economic burden of HAIs that are identified after discharge. For example, surgical site infections ${ }^{24,25}$ and hospital-acquired Clostridium difficile infections ${ }^{26-29}$ often manifest after hospital discharge.

An important limitation to this study is publication bias. The estimates of time-dependent bias that we report come from published studies, many of which were focused on emphasizing the new methods for handling this bias. We were 
unable to find any published studies that reported zero or negative time-dependent bias. Furthermore, time-dependent bias could be less relevant in analyses of excess LOS after surgical site infection in patients with infection onset after discharge.

In conclusion, treating HAI as a time-fixed variable, as opposed to a time-varying variable, leads to substantial inflation of the effect of HAI on excess LOS. Future studies aiming to determine the excess costs and LOS of HAI should utilize one of several methods we discussed to reduce time-dependent bias and lead to more accurate estimates of HAI outcomes.

\section{ACKNOWLEDGMENTS}

Financial support: The work in this paper was conducted through a contract with the Centers for Disease Control and Prevention. This material is the result, in part, of work supported with resources and the use of facilities of the George E. Wahlen Department of Veterans Affairs Medical Center, Salt Lake City, Utah.

Potential conflicts of interest: All authors report no conflicts of interest relevant to this article.

Address correspondence to Richard E. Nelson, PhD, 500 Foothill Blvd, Salt Lake City, UT 84148 (richard.nelson@utah.edu).

\section{REFERENCES}

1. Brown J, Doloresco Iii F, Mylotte JM. "Never events": not every hospital-acquired infection is preventable. Clin Infect Dis 2009;49:743-746.

2. Selected Medicare hospital quality provisions under the ACA. American Association of Medical Colleges website. https://www. aamc.org/advocacy/medicare/153882/selected_medicare_hospital_ quality_provisions_under_the_aca.html. Published June 8, 2010. Accessed November 6, 2014.

3. Barnett AG, Beyersmann J, Allignol A, Rosenthal VD, Graves N, Wolkewitz M. The time-dependent bias and its effect on extra length of stay due to nosocomial infection. Value Health 2011;14:381-386.

4. Samore M, Harbarth S. A Methodologically focused review of the literature in hospital epidemiology and infection control. In: Mayhill CG, ed. Hospital Epidemiology and Infection Control. Philadelphia: Lippincott Williams \& Wilkins, 2004.

5. Beyersmann J, Gastmeier P, Wolkewitz M, Schumacher M. An easy mathematical proof showed that time-dependent bias inevitably leads to biased effect estimation. J Clin Epidemiol 2008;61:1216-1221.

6. van Walraven C, Davis D, Forster AJ, Wells GA. Time-dependent bias was common in survival analyses published in leading clinical journals. J Clin Epidemiol 2004;57:672-682.

7. De Angelis G, Murthy A, Beyersmann J, Harbarth S. Estimating the impact of healthcare-associated infections on length of stay and costs. Clin Microbiol Infect 2010;16:1729-1735.

8. Cosgrove SE, Kaye KS, Eliopoulous GM, Carmeli Y. Health and economic outcomes of the emergence of third-generation cephalosporin resistance in Enterobacter species. Arch Intern Med 2002;162:185-190.

9. Jiang Y, Viner-Brown S, Baier R. Burden of hospital-onset Clostridium difficile infection in patients discharged from Rhode
Island hospitals, 2010-2011: application of present on admission indicators. Infect Control Hosp Epidemiol 2013;34:700-708.

10. Sammons JS, Localio R, Xiao R, Coffin SE, Zaoutis T. Clostridium difficile infection is associated with increased risk of death and prolonged hospitalization in children. Clin Infect Dis 2013; 57:1-8.

11. Stewart DB, Hollenbeak CS. Clostridium difficile colitis: factors associated with outcome and assessment of mortality at a national level. J Gastrointest Surg 2011;15:1548-1555.

12. Wolkewitz M, Beyersmann J, Gastmeier P, Schumacher M. Efficient risk set sampling when a time-dependent exposure is present: matching for time to exposure versus exposure density sampling. Methods Inf Med 2009;48:438-443.

13. Schulgen G, Kropec A, Kappstein I, Daschner F, Schumacher M. Estimation of extra hospital stay attributable to nosocomial infections: heterogeneity and timing of events. J Clin Epidemiol 2000;53:409-417.

14. Roberts RR, Scott RD, 2nd, Hota B, et al. Costs attributable to healthcare-acquired infection in hospitalized adults and a comparison of economic methods. Med Care 2010;48:1026-1035.

15. De Angelis G, Allignol A, Murthy A, et al. Multistate modelling to estimate the excess length of stay associated with meticillinresistant Staphylococcus aureus colonisation and infection in surgical patients. J Hosp Infect 2011;78:86-91.

16. Wolkewitz M, Allignol A, Harbarth S, de Angelis G, Schumacher M, Beyersmann J. Time-dependent study entries and exposures in cohort studies can easily be sources of different and avoidable types of bias. J Clin Epidemiol 2012;65:1171-1180.

17. Macedo-Vinas M, De Angelis G, Rohner P, et al. Burden of meticillin-resistant Staphylococcus aureus infections at a Swiss University hospital: excess length of stay and costs. J Hosp Infect 2013;84:132-137.

18. Schumacher M, Allignol A, Beyersmann J, Binder N, Wolkewitz M. Hospital-acquired infections-appropriate statistical treatment is urgently needed! Int J Epidemiol 2013;42:1502-1508.

19. Vrijens F, Hulstaert F, Van de Sande S, Devriese S, Morales I, Parmentier Y. Hospital-acquired, laboratory-confirmed bloodstream infections: linking national surveillance data to clinical and financial hospital data to estimate increased length of stay and healthcare costs. J Hosp Infect 2010;75:158-162.

20. Vrijens F, Hulstaert F, Devriesse S, van de Sande S. Hospitalacquired infections in Belgian acute-care hospital: an estimation of their global impact on mortality, length of stay and healthcare costs. Epidemiol Infect 2012;140:126-136.

21. Suissa S. Immortal time bias in pharmaco-epidemiology. Am J Epidemiol 2008;167:492-499.

22. Samore $\mathrm{MH}$, Shen $\mathrm{S}$, Greene $\mathrm{T}$, et al. A simulation-based evaluation of methods to estimate the impact of an adverse event on hospital length of stay. Med Care 2007;45:S108-S115.

23. Fact sheets: CMS final rule to improve quality of care during hospital inpatient stays. Centers for Medicare and Medicaid Services website. http://www.cms.gov/newsroom/mediareleasedatabase/ fact-sheets/2013-fact-sheets-items/2013-08-02-3.html. Published 2013. Accessed November 7, 2014.

24. McIntyre LK, Warner KJ, Nester TA, Nathens AB. The incidence of post-discharge surgical site infection in the injured patient. J Trauma 2009;66:407-410.

25. Sands K, Vineyard G, Platt R. Surgical site infections occurring after hospital discharge. J Infect Dis 1996;173:963-970. 
26. Chang HT, Krezolek D, Johnson S, Parada JP, Evans CT, Gerding DN. Onset of symptoms and time to diagnosis of Clostridium difficile-associated disease following discharge from an acute care hospital. Infect Control Hosp Epidemiol 2007;28: 926-931.

27. Dial S, Kezouh A, Dascal A, Barkun A, Suissa S. Patterns of antibiotic use and risk of hospital admission because of Clostridium difficile infection. CMAJ 2008;179:767-772.
28. Dubberke ER, McMullen KM, Mayfield JL, et al. Hospitalassociated Clostridium difficile infection: is it necessary to track community-onset disease? Infect Control Hosp Epidemiol 2009;30:332-337.

29. Palmore TN, Sohn S, Malak SF, Eagan J, Sepkowitz KA. Risk factors for acquisition of Clostridium difficile-associated diarrhea among outpatients at a cancer hospital. Infect Control Hosp Epidemiol 2005;26:680-684. 